\title{
Properties of the extremely HI-massive galaxy HIZOA J0836-43
}

\section{Renée C.Kraan-Korteweg*}

Astronomy Department, University of Cape Town

E-mail: kraaneast.uct.ac.za

\section{Michelle E. Cluver}

IPAC, California Institute of Technology

E-mail: mcluvereipac.caltech.edu

\section{Tom H. Jarrett}

IPAC, California Institute of Technology

E-mail: jarretteipac.caltech.edu

\section{Patrick A. Woudt}

Astronomy Department, University of Cape Town

E-mail: pwoudteast.uct.ac.za

\begin{abstract}
Little is known about the properties of extremely massive HI-galaxies. They are extremely scarce and are - according to hierarchical structure formation - only forming now $(z<1)$. The forthcoming deep HI SKA Pathfinders surveys will uncover many more of them. This will lead to a better understanding of their evolution and frequency, and the shape of the bright end of the HI mass function.

The recently discovered galaxy HIZOA J0836-43 is one of the most HI-rich galaxies $\left(M_{\mathrm{HI}}=7.5 \times 10^{10} \mathrm{M}_{\odot}\right)$ - and the nearest of its kind. As such it is an ideal local probe of these elusive galaxies. Results from a detailed investigation in the near- (IRSF) and far-infrared (Spitzer) of this local HI-massive galaxy are presented. Unlike other giant HI galaxies, it is not of low surface brightness. The galaxy is found to be a luminous starbursting galaxy at an unexpected early stage of stellar mass building, more typical of star-forming galaxies at higher redshift $(z \sim 0.7)$. With regard to its environment, hence possible clues to its formation, the near infrared imaging survey finds HIZOA J0836-43 to lie in a region underdense in $L^{*}$ galaxies - consistent with the observation that HI-massive galaxies are preferentially found in low density regions - in the presence, however, of an uncommonly large number of low stellar mass galaxies.
\end{abstract}

Panoramic Radio Astronomy: Wide-field 1-2 GHz research on galaxy evolution

June 2-5 2009

Groningen, the Netherlands

\footnotetext{
* Speaker.
} 


\section{Introduction}

The number of galaxies with HI-masses above a characteristic value, $M_{\mathrm{HI}}^{*}=6 \cdot 10^{9} \mathrm{M}_{\odot}$ declines exponentially (Zwaan et al. 2005). The most massive galaxies are very scarce, their number density is ill-constrained, and little is known about their properties.

Hierarchical structure formation predicts these galaxies to be forming only recently ( $z<1$; Mo et al. 1998) although models overpredict their current population ( $z=0-1$; Renzini 2007). Local massive and gas-rich galaxies are thus expected to be in an early stage of formation. But the very few known ones - like Malin 1 (Impey \& Bothun 1989) - are rather quiescent, providing few clues about their past or future evolution.

A detailed investigation of the recently discovered, fairly nearby $\left(v_{h e l}=10689 \mathrm{~km} \mathrm{~s}^{-1}\right)$ galaxy HIZOA J0843-36 (Donley et al. 2006), one of the most massive HI-galaxies known to date, will therefore provide a valuable local probe to be put into context with the forthcoming higher redshift pathfinder surveys.

HIZOA J0843-36 was discovered with the Parkes radio telescope in the deep systematic HI survey of the Zone of Avoidance (ZOA; e.g. Kraan-Korteweg et al. 2005). Follow-up ATCA and AAT radio observations confirmed it to be amongst the most massive spiral galaxies (Donley et al. 2006). It contains $7.5 \times 10^{10} \mathrm{M}_{\odot}$ of $\mathrm{HI}$ gas, has a total dynamical mass of $1.4 \times 10^{12} \mathrm{M}_{\odot}$ and a 20 -cm derived star formation rate of $\sim 35 \mathrm{M}_{\odot} / \mathrm{yr}$. It also has a prominent bulge in the near-infrared (bulge-to-disk ratio in the $K_{s}$ band of $\sim 0.80$ ) which lies central to its enormous, rapidly rotating HI disk. It is amongst the most HI-massive spiral galaxies known to date and, at a distance of 148 Mpc, also the nearest. Contrary to other HI-rich galaxies, like Malin 1, it is, however, not a giant low surface brightness galaxy.

This leads to the question: What is happening in this unusual galaxy? Is the galaxy undergoing a starburst, or is it an AGN? Can its environment provide clues about its origin/formation? How did it grow to its current size? Was it through merger/accretion processes?

\section{Near and Mid-Infrared observations of HIZOA J0836-43}

A detailed investigation of HIZOA J0843-3 is rendered difficult due to its location behind the Vela supernova remnant in the ZOA. Lying behind a dust layer of $A_{B}=10 \mathrm{~m} 0$ it is optically invisible. To minimize the effects of foreground extinction, we launched an observational campaign in the infrared of the galaxy and its local environment. The program comprises imaging of an area of $28^{\prime} \times 28^{\prime}$, centered on the galaxy, obtained by using Spitzer with IRAC $(3.6,4.5,5.8,8.0 \mu \mathrm{m})$ and MIPS $(24.0,70,160 \mu \mathrm{m})$, as well as IRS high and low resolution spectroscopy of the galaxy itself. We also performed a deep imaging survey $\left(\mathrm{JHK}_{S}\right)$ of $1.5 \times 1.5$ with the Infrared Survey Facility (IRSF) at the $1.4 \mathrm{~m}$ Japanese telescope in Sutherland (SAAO), see Cluver (2009) for details.

\subsection{Infrared properties of HIZOA J0836-43}

The near-infrared light profiles of the galaxy appear typical for an early spiral or lenticular with its prominent bulge, while the radio observations suggest recent, active star formation. The new data find a NIR - MIR Spectral Energy Distribution that is consistent with an Sc morphology (from GRASIL modeling). However, the total IR luminosity of $L_{\mathrm{TIR}}=1.2 \times 10^{11} \mathrm{~L} \odot$ and star-formation 
rate in the far infrared (FIR) of $\sim 21 \mathrm{M}_{\odot} / \mathrm{yr}$ is much higher than that of typical disk spirals and is more consistent with the properties of local Luminous Infrared Galaxies (LIRGs). The galaxy also has substantial cold molecular gas $\left(M_{\mathrm{H}_{2}}=1.3 \times 10^{10} \mathrm{M}_{\odot}\right)$ as estimated from the FIR, but little warm dust. The galaxy possesses a prominent bulge of evolved stars and a total stellar mass of $4.4 \times 10^{10} \mathrm{M}_{\odot}$ (Cluver et al. 2009).

In summary, we have a starbursting galaxy (the spectrum shows no signs of AGN activity) with a prominent bulge comprised of evolved stars. But its specific star formation rate $\left(\sim 0.5 \mathrm{Gyr}^{-1}\right)$ implies an early stage of stellar mass building. With its huge reservoir reservoir of gas it could double its stellar mass in about 2 Gyr. Based on the stellar surface brightness profile and emission from tracers of star formation, the galaxy seems to be undergoing inside-out star formation similar to processes observed at higher redshift $(z \sim 0.7)$ where gas fractions of disks were higher compared to local galaxies (e.g. Bell et al. 2005, Pérez-González et al. 2005). Despite its extreme H I mass, both the near- and mid-infrared luminosity appear consistent with expectations from $\mathrm{H} \mathrm{I} /$ luminosity relations in these bands. This suggests relatively "normal" evolution in HIZOA J0836-43, despite lying at the extreme high end of the relation.

\subsubsection{Environment of HIZOA J0836-43}

The question that remains unanswered is: did HIZOA J0843-36 grow to its current size through cannibalism in a dense environment or accretion along filaments? Early NIR observations found two possible companions, further supported by the subsequent Spitzer observations.

The deep NIR IRSF survey of $2.24 \square^{\circ}$ around the massive galaxy revealed 404 galaxies, most previously unknown. Of the 44 MASX object in the survey area, only 16 coincided with our galaxies. The deeper and higher resolution IRSF images found the 28 other 2MASX objects to either be blended (unresolved) star images or Galactic objects. According to photometric redshifts (Jarrett et al. 2009, in prep.), only a handful of the IRSF galaxies lie at the distance of the massive galaxy.

The absolute magnitude distribution of the galaxies that are - according to their photometric redshifts - located within a volume of radius $r=10 \mathrm{Mpc}$ around HIZOA J0836-43 are compared to four regions of varying galaxy density at similar redshifts. This includes a field sample, a supercluster filament, a fork apex, and a cluster (Hercules). The comparison reveals that the population of galaxies in the surroundings of the massive galaxy is constituted of an overdensity of low stellar mass galaxies $\left(M_{K_{s}}^{o} \gtrsim-23\right.$. 0 ) with only a handful of brighter galaxies. Even fewer than in the comparison field sample.

Hence, the massive galaxy HIZOA J0836-43 lives in a low $L^{*}$ density environment. That may have allowed its formation and survival, enabling it to evolve into the unusual LIRG starburst galaxy we observe today. It also seems consistent with the observation that HI-massive galaxies are preferentially found in low density regions.

Inspection of the global larger-scale structures in which HIZOA J0836-43 is embedded finds this galaxy to lie in a slightly enhanced density of fainter galaxies within a (newly identified) filamentary structure encircling a void (Cluver 2009). The conditions appear favorable for the galaxy to have grown this large HI disk, both through accretion of gas due to minor merging, as well as infall of gas along the filament. 


\section{Next Steps}

This is the only known HI-massive galaxy undergoing an infrared-luminous starburst. It appears to be in the process of building a disk, fueled by its large gas reservoir. We have obtained millimeter observations with MOPRA to complement the infrared study of the galaxy's star formation phase and have detected the very broad CO (1-0) line of HIZOA J0836-43. We find a total molecular gas mass $\left(\mathrm{H}_{2}+\right.$ Helium, where the He fraction is 1.38$)$ of $4.5 \times 10^{9} \mathrm{M}_{\odot}$ corresponding to a $\mathrm{H}_{2}$-to-HI gas-mass fraction of $\sim 6 \%$. We, however, require deeper observations to obtain the sensitivity to detect the low velocity $\mathrm{CO}$ gas and map the extent of molecular gas in the disk. This will provide clues regarding the galaxy's past and present evolution.

A future deep HI survey with MeerKAT (or ASKAP) will shed further light on the environment of the galaxy with its association of low stellar mass, but possibly HI-rich galaxies. Previous simulations of MeerKAT performance in the ZOA in the Great Attractor region (Kraan-Korteweg et al. 2009) have proven its effectiveness in answering these questions on reasonable timescales.

Such an HI-survey will furthermore give substance to the findings by Kraan-Korteweg (in prep.) of the existence of a massive overdensity lurking in the ZOA in Vela at $v_{\text {hel }} \sim 20000 \mathrm{~km} \mathrm{~s}^{-1}$. This overdensity is a complete surprise. Some suggestions of its existence are seen in the reconstructed density maps based on the MASX Redshift Survey (Erdogdu et al. 2006). If confirmed, this would have major implications for the the dynamics in the nearby Universe. It may well help explain the large-scale flows towards that direction found by Hudson et al. (2009).

Acknowledgment: Financial support from the National Research Foundation and the University of Cape Town is gratefully acknowledged.

\section{References}

[1] Bell E.F., Papovich C., Wolf C. et al. 2005, ApJ 625, 23

[2] Cluver M.E. 2009, PhD thesis, University of Cape Town

[3] Cluver M.E., Jarrett T.H., Appleton P.N., Kraan-Korteweg R.C. et al. 2008, ApJ 6 98, L17

[4] Donley J.L., Koribalski B.S., Staveley-Smith L., et al. 2006, MNRAS 369, 1741

[5] Erdogdu P., Lahav O., Huchra J.P. et al. 2006, MNRAS 373, 45

[6] Hudson M.J., Smith R.J., Lucey, J.R., Branchini E. 2009, MNRAS 352, 61

[7] Impey C., Bothun G. 1989, ApJ 341, 89

[8] Kraan-Korteweg R.C., Staveley-Smith, L., Donley J.L. et al. 2005, IAU Symp. 216, 203

[9] Kraan-Korteweg R.C., van der Heyden K.J., Cluver M.E., Woudt P.A. 2009, First MEARIM, eds. A. Hady \& M.J. Wamas, Cairo University Press, 27 - 34 (arXiv0810.3929)

[10] Meyer M.J., Zwaan, M.A., Webster R.L. et al. 2004, MNRAS 350, 1195

[11] Mo H.J., Mao S., White S.D.M. 1998, MNRAS 295, 319

[12] Pérez-González, P.G., Rieke, G.H., Egami, E. et al., 2005, ApJ , 630, 82.

[13] Renzini A. 2007, ASP CS 380, 309

[14] Zwaan M.A., Meyer M.J., Staveley-Smith L., Webster R.L. 2005, MNRAS 359, L30 